\title{
"INTERMITTENT" CHAOS AND SUBHARMONICS IN SWITCHING POWER SUPPLIES
}

\author{
Chi K. Tse, Yufei Zhou ${ }^{\dagger}$ Francis C. M. Lau \\ Dept. of Electronic \& Information Engineering \\ Hong Kong Polytechnic University, Hong Kong \\ ${ }^{\dagger}$ Also with Anhui University, Hefei, China
}

\begin{abstract}
This paper studies the "intermittent" chaos and subharmonics observed in switching $\mathrm{dc} / \mathrm{dc}$ converters using a simple circuit model that describes possible unintended coupling of some weak spurious signal to the converter. The study shows that the signal strength and frequency of the intruding signal are vital parameters that affect the type of intermittent behaviour and the period of intermittency. Simulations and experimental results are presented.

Index Terms - Switching converters, spurious signal coupling, "intermittent" chaos.
\end{abstract}

\section{INTRODUCTION}

It is not uncommon in the design of switching power supplies that the rather puzzling irregular (chaotic) behaviour is observed intermittently between long periods of regular behaviour. Noise or bad construction is the usual blame. In this paper a simple circuit model is proposed to explain the "intermittent" chaos observed in switching dc/dc converters. The model essentially incorporates a weak periodic signal which is coupled to the converter via unintended paths (e.g., conducted or radiated EMI). The study shows that the signal strength and frequency of the intruding signal are vital parameters that affect the type of intermittent behaviour and the period of intermittency.

\section{SIMPLIFIED MODEL OF SPURIOUS SIGNAL INTRUSION}

We begin with the crude but practically valid assumption that the power supply under study is not perfectly protected from intrusion of signals which are generated outside the power supply [1]. The intrusion can take the form of coupling via conducted or radiated paths. Sometimes, the intruders can live on the same circuit board or be present at a very close proximity. Figure 1 (a) shows a crude model which illustrates the situation. In order to study the effects of coupling of spurious (intruding) signals, we need a circuit model that describes the way in which the power supply

\author{
Shui-Sheng Qiu
}

\author{
College of Electronic \& Information Engineering \\ South China University of Technology \\ Guangzhou, China
}

is connected to the spurious signal source. One simple approach is to model the coupling as an additive process which injects the spurious signal directly to the reference voltage of the power supply, as shown in Fig. 1 (b).

Suppose the effective additive source is $v_{s}$, which may be due to some oscillators or PWM generators present in the proximity. For convenience we denote the strength of the spurious source by $\alpha$ which is the ratio of the amplitude

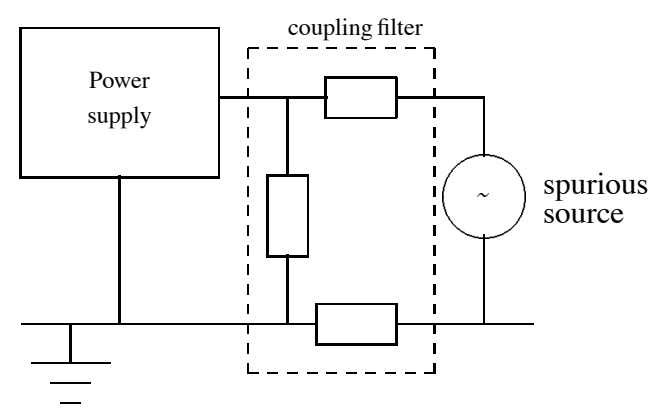

(a)

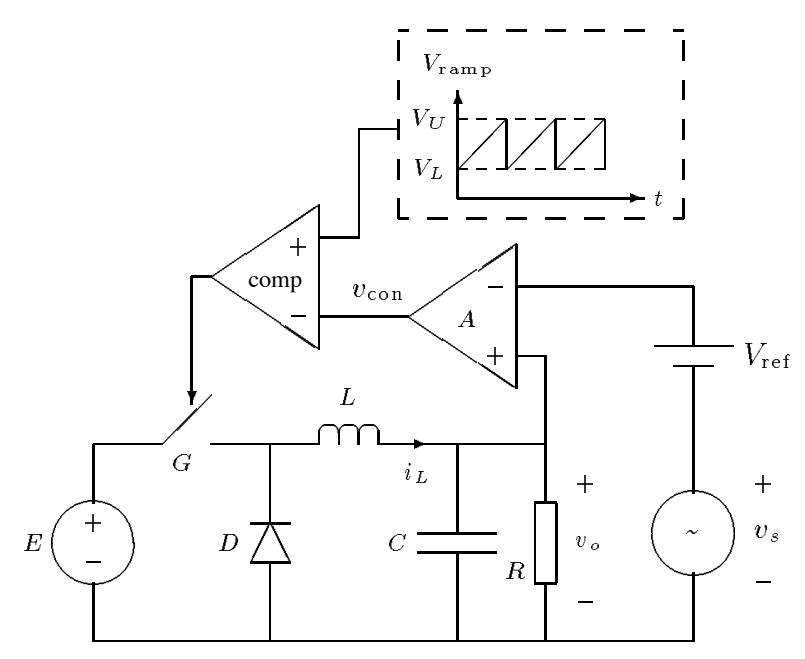

(b)

Fig. 1: A power supply coupled with spurious source. (a) Crude model; (b) circuit model showing a buck converter with an additive intruding signal $v_{s}$ acted upon the reference voltage. 

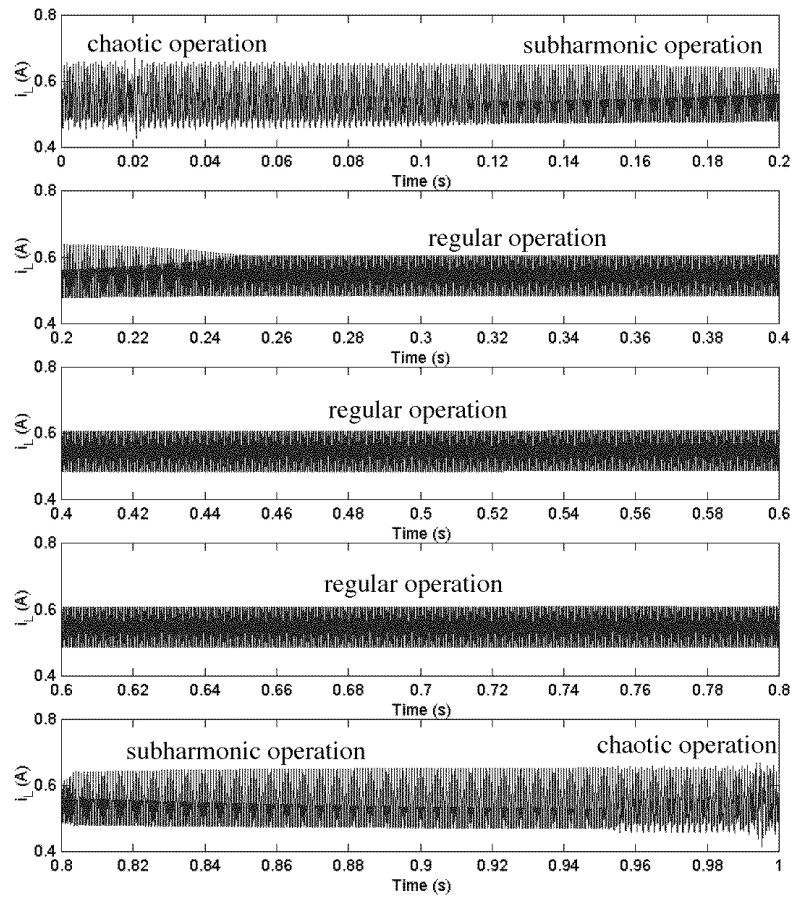

(a)
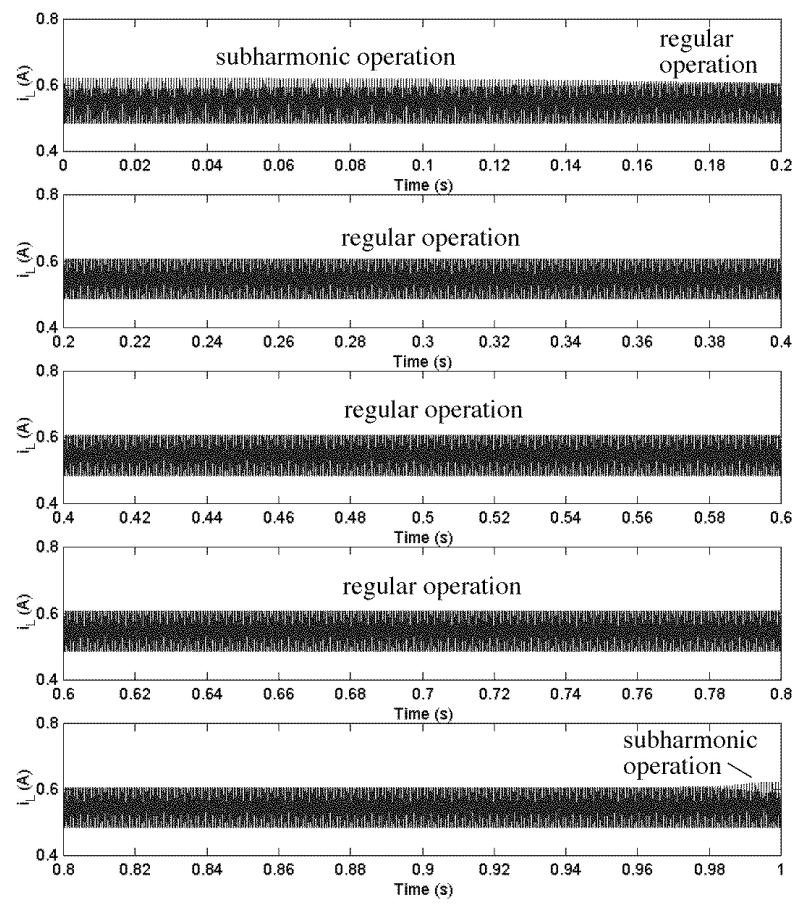

(b)

Fig. 2: Inductor current waveform for buck converter with unintended coupling of sinusoidal intruding source for (a) $\alpha=0.0034$ showing "intermittent" chaos, and (b) $\alpha=0.0003$ showing "intermittent" subharmonics.

of $v_{s}$ to $V_{\text {ref }}$, i.e.,

$$
\alpha=\hat{v}_{s} / V_{\text {ref }}
$$

where $\hat{v}_{s}$ is amplitude of the effective intruding source appeared behind the reference voltage of the power supply. We will investigate the effect of coupling the intruding source to the power supply for various levels of strengths of the intruding source.

\section{QUICK GLIMPSE OF INTERMITTENT CHAOS}

When $v_{s}$ is a sinusoidal signal of frequency $f_{s}$, the reference voltage is being modulated as

$$
V_{\mathrm{ref}} \mapsto V_{\mathrm{ref}}\left(1+\alpha \sin 2 \pi f_{s} t\right)
$$

Such spurious coupling can occur in power supplies that operate in an RF environment. Suppose the switching frequency is $f_{o}$, and $f_{0} \neq f_{s}$. Obviously, we would expect the effect of the intruding source on the power supply to be periodic in $1 /\left|f_{0}-f_{s}\right| .^{1}$

To get a quick glimpse of the phenomenon, let us consider the buck converter shown in Fig. 1 (a) for two particular effective signal strengths. The circuit parameters are as follows: $L=20 \mathrm{mH}, C=47 \mu \mathrm{F}, R=22 \Omega$,

\footnotetext{
${ }^{1}$ We have also studied the case where the intruding signal is a rectangular pulse. The analysis and results are omitted here due to space limitation.
}

$f_{0}=2500 \mathrm{~Hz}, E=24 \mathrm{~V}, V_{L}=3.8 \mathrm{~V}, V_{U}=8.2 \mathrm{~V}$ and $V_{\text {ref }}=11 \mathrm{~V}$. The spurious signal frequency $f_{s}$ is $2501 \mathrm{~Hz}$. Time-domain cycle-by-cycle simulations are performed and the inductor current waveform is shown in Fig. 2. Here, we observe "intermittent" chaos and "intermittent" subharmonics for relatively strong and weak coupling, respectively. ${ }^{2}$

These kinds of phenomena are commonly observed by practicing power supply engineers. Here, we show that the phenomena can be explained in terms of coupling of a spurious signal through unintended paths to the power supply.

The intermittent period is equal to the difference between the intruding signal frequency and the power supply's switching frequency. In this case, it is 1 second.

\section{TIME-BIFURCATION DIAGRAMS}

For a clear exposition of the phenomenon, it is customary in the systems theory literature to examine the "sampled waveforms", which effectively ignore the detailed switching ripples but focus on the movement of the waveforms at periodic switching instants. Specifically we sample the waveforms at $t=n T$, where $T$ is the switching period,

\footnotetext{
${ }^{2}$ The term intermittent chaos has been used in the mathematics literature to describe a few special types of chaotic behaviour [2]. Here, we use the term "intermittent" chaos to describe a particular operation where chaos shows up periodically between regular operations. Such intermittent operation is sometimes called breathing in the physics literature [3].
} 


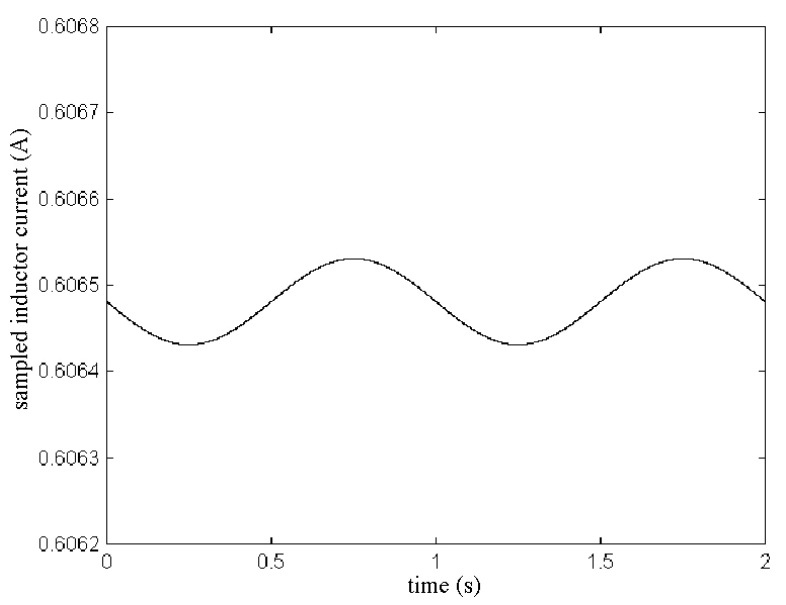

(a) $\alpha=0.0001$

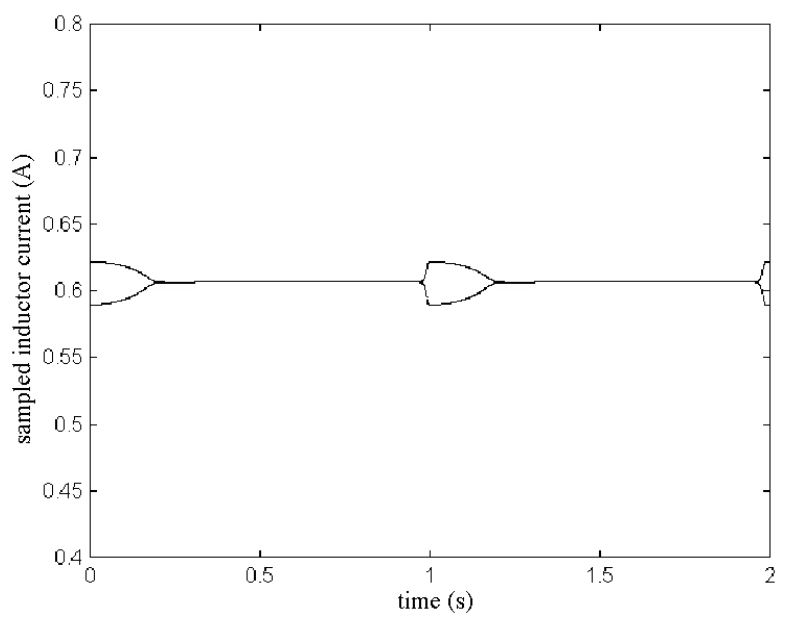

(c) $\alpha=0.0003$

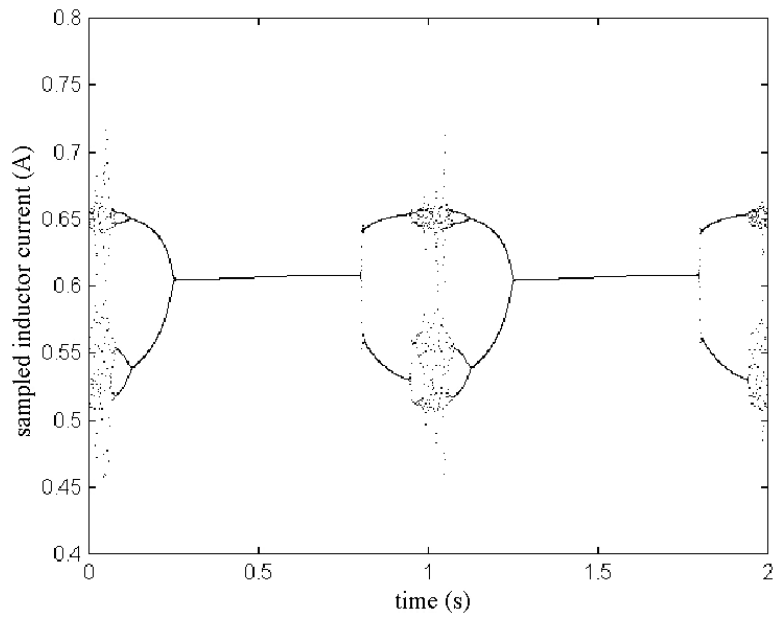

(e) $\alpha=0.0034$



(b) $\alpha=0.00022$

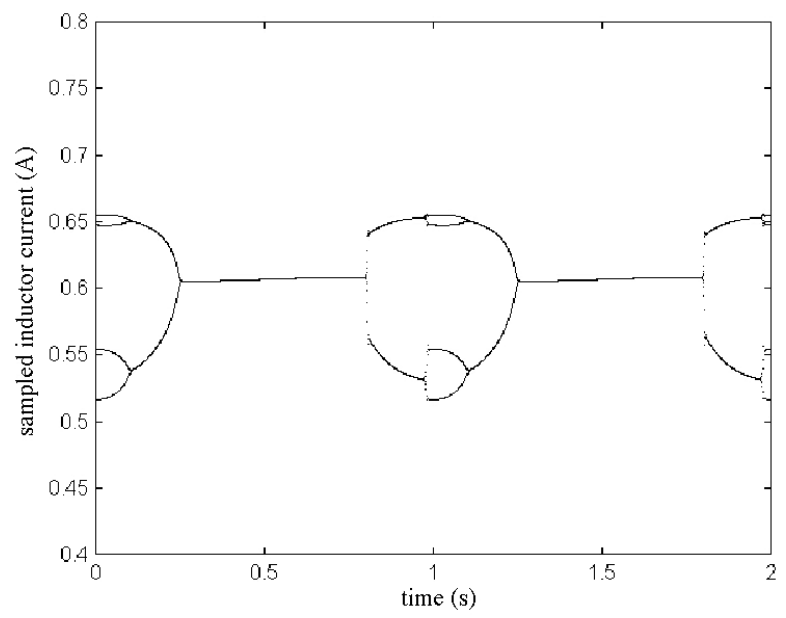

(d) $\alpha=0.0031$



(f) $\alpha=0.007$

Fig. 3: Sampled inductor current waveforms (time-bifurcation diagrams) for buck converter with unintended coupling of sinusoidal intruding source for different coupling strengths. (a) Regular operation with fluctuation of average value; (b)-(d) "intermittent" subharmonics; (e)-(f) "intermittent" chaos. 
i.e., $T=1 / f_{o}$, and the resulting plots are called timebifurcation diagrams since they reflect the change of qualitative behaviour as time goes.

We now consider the case of sinusoidal intruding signals as described in the previous section, i.e.,

$$
v_{s}=\alpha V_{\mathrm{ref}} \sin 2 \pi f_{s} t
$$

Some typical time-bifurcation diagrams are shown in Fig. 3.

When the intruding signal strength is very weak, the power supply can still maintain its regular operation, though the average operating point fluctuates. The effect is not significant. As the intruding signal strength increases, the power supply experiences subharmonic operation intermittently with regular operation. For a relatively low intruding signal strength, period-2 subharmonics are observed intermittently with regular operation. Further increase in intruding signal strength causes period-4 subharmonics to occur intermittently with period-2 subharmonics and regular operation. When the intruding signal strength is strong, the power supply experiences chaotic operation intermittently with subharmonic operation and regular operation. The intermittent period is equal to the $1 /\left|f_{0}-f_{s}\right|$. Thus, if the intruding signal frequency is very close to the switching frequency of the power supply, the intermittency is long. ${ }^{3}$

\section{EXPERIMENTAL OBSERVATIONS}

We have built a circuit prototype to emulate the proposed coupling circuit. The circuit parameters are the same as those used in the simulations. The intruding signal is a sinusoidal source. By varying the intruding signal strength, we observe "intermittent" subharmonics and chaos consistent with the simulations. Figure 4 shows a few time-bifurcation diagrams obtained experimentally.

\section{CONCLUSIONS}

Power electronics are nonlinear systems which have been shown to exhibit a variety of complex behavior [4]. In this paper we attempt to rationalize a commonly observed but rarely explained phenomenon in power supply design. By using an appropriate circuit model, "intermittent" chaos and subharmonics can be explained in terms of coupling of spurious signals. This paper shows that the signal strength and frequency of the intruding signal are vital parameters that affect the type of intermittent behaviour and the period of intermittency.

\footnotetext{
${ }^{3}$ Limited by space, we omit the results for the case where the intruding signal is a rectangular pulse. Nonetheless, the general pattern is similar.
}

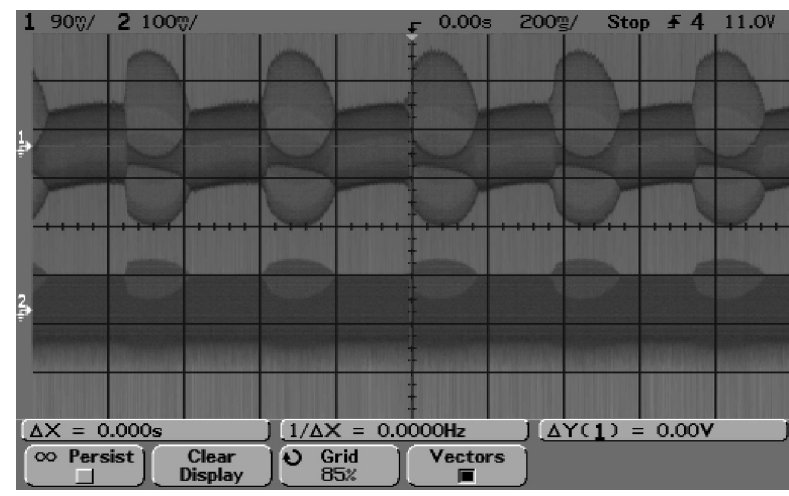

(a) $\alpha=0.0012$ (upper $90 \mathrm{mV} /$ div; lower $100 \mathrm{~mA} / \mathrm{div}$ )

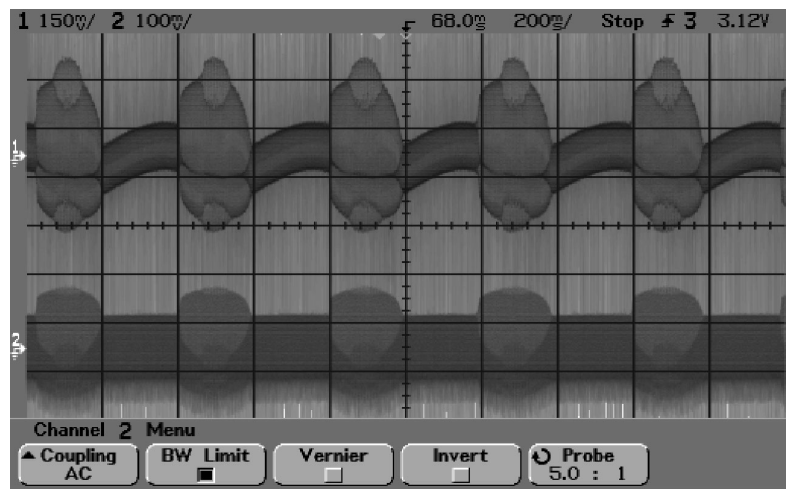

(b) $\alpha=0.0036$ (upper $150 \mathrm{mV} /$ div; lower $100 \mathrm{~mA} / \mathrm{div}$ )

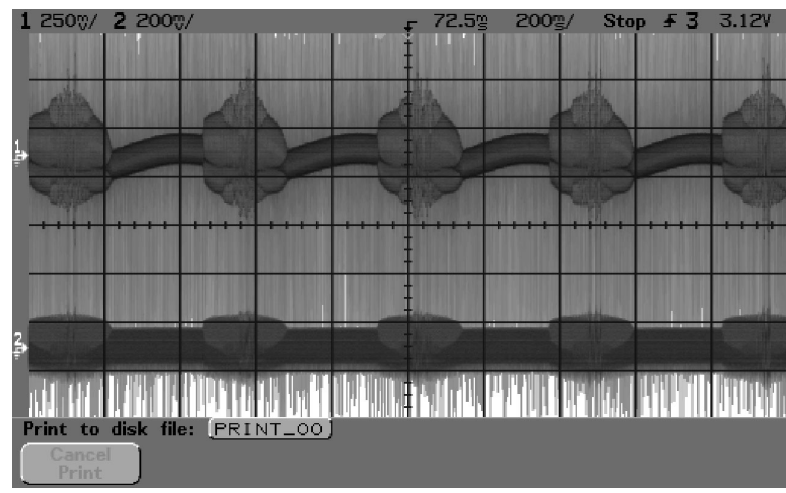

(c) $\alpha=0.004$ (upper $250 \mathrm{mV} /$ div; lower $200 \mathrm{~mA} / \mathrm{div}$ )

Fig. 4: Measured time-bifurcation diagrams. Upper trace: output voltage; lower trace: inductor current. (a) "Intermittent" period2 subharmonics; (b) "intermittent" period-2 and period-4 subharmonics; (c) "intermittent" chaos.

\section{REFERENCES}

[1] J.A. Ferreira, P.R. Willcock, S.R. Holm, "Sources, paths and traps of conducted EMI in switch mode circuits," Proc. IEEE Ind. Appl. Conf., pp. 1584-1591, 1997.

[2] E. Ott, Chaos in Dynamical Systems, CUP, 1993.

[3] Z. Qu, G. Hu, G. Yang, G. Qin, "Phase effect in taming nonautonomous chaos by weak harmonic perturbations," Phys. Rev. Lett. 74(10), pp. 1736-1739, 1995.

[4] C.K. Tse and M. di Bernardo, "Complex behavior in switching power converters," Proceedings of IEEE, 90(5), pp. 768-781, 2002. 